\title{
Synthesis, characterization and conductivity studies of polypyrrole-fly ash composites
}

\author{
M V MURUGENDRAPPA ${ }^{\dagger}$, SYED KHASIM ${ }^{\ddagger}$ and M V N AMBIKA PRASAD* \\ Department of Materials Science and Physics, Gulbarga University, Gulbarga 585 106, India \\ ${ }^{\dagger}$ Department of Physics, GM Institute of Technology, Davangere 577 006, India \\ Department of Physics, KBN College of Engineering, Gulbarga 585 104, India
}

MS received 10 January 2005; revised 5 May 2005

\begin{abstract}
In situ polymerization of pyrrole was carried out in the presence of fly ash (FA) to synthesize polypyrrole-fly ash composites (PPy/FA) by chemical oxidation method. The PPy/FA composites have been synthesized with various compositions $(10,20,30,40$ and $50 \mathrm{wt} \%)$ of fly ash in pyrrole. The surface morphology of these composites was studied with scanning electron micrograph (SEM). The polypyrrole-fly ash composites were also characterized by employing X-ray diffractometry (XRD) and infrared spectroscopy (IR). The a.c. conductivity behaviour has been investigated in the frequency range $10^{2}-10^{6} \mathrm{~Hz}$. The d.c. conductivity was studied in the temperature range from $40-200^{\circ} \mathrm{C}$. The dimensions of fly ash in the matrix have a greater influence on the observed conductivity values. The results obtained for these composites are of greater scientific and technological interest.
\end{abstract}

Keywords. Polypyrrole; fly ash; composites; conductivity.

\section{Introduction}

The electrical transport in polymeric materials (Kivelson 1981; Singh et al 1991) has become an area of increasing interest in research because these materials possess a great potential for solid state devices. Conducting polymer composites have attracted considerable interest in recent years because of their numerous applications in variety of electric and electronic devices. It has been found that such composites can exhibit some novel properties. Polypyrrole has been regarded as one of the most studied conducting polymers (Omastova 1993). The understanding of electrical properties, morphology and crystal structure of polypyrrole composites may be useful in improving the stability characteristics of these materials which are the key factors in governing the device performance.

One way of making these composites involves synthesizing the conducting polymer inside the matrices of conventional polymers using chemical (Mohammadi et al 1993) or electrochemical (Niwa and Tamamura 1984) polymerization. There are many reports pertaining to the chemical preparation of conducting polymer composites without using any insulating polymer (Meadea and Armes 1995). The most preferred method for synthesis of PPy composites is to use ammonium persulfate as an oxidant.

Fly ash is a waste product produced from coal fired thermal power stations during the combustion of coal. It

*Author for correspondence (Prasad1_Amb@rediffmail.com) is an alkaline grey powder with $\mathrm{pH}$ ranging from 9-9.9. Large number of coal fired power plants all over the world dispose a large quantity of fly ash causing serious environmental problems (Sahu 1994). Less than half of the ash is used as a raw material for concrete manufacturing and construction; the remaining is directly dumped on land side as land fill or simply piled up. Due to environmental regulations, new ways of utilizing FA have to be explored in order to safeguard the environment and provide useful ways for its disposal. Hence, there is considerable interest in utilization of FA as raw material. For the first time, FA was used in the preparation of cordierite (Sampathkumar et al 1995). Because of the presence of $\mathrm{SiO}_{2}$ and $\mathrm{Al}_{2} \mathrm{O}_{3}$ in high proportions, the FA was used to synthesize zeolites (LaRosa et al 1992). FA was treated hydrothermally and the performance of this material as cracking catalyst was investigated with heavy oil fraction as the cracking feed stock (Ojha and Pradhan 2001). On the other hand, there were many experimental analyses on FA to undertake basic compositional, physical and chemical properties for technical studies and applications (Cumpston et al 1992). Raw fly ash consists of quartz and mullite as crystalline phases and some quantity of glassy phase (Raghavendra et al 2002a). Effort has been made to understand the electrical conductivity and dielectric behaviour of fly ash and it was observed that these materials possess very high relative dielectric constant of the order of $10^{4}$. Such a high dielectric constant is one of the important parameters in capacitor fabrication, and microwave absorption applications. Through this work 
we have made a successful effort in preparing polypyrrole-fly ash composites, which gives newer ways of better utility of fly ash.

Conducting polymer composites with some suitable compositions of one or more insulating materials lead to desirable properties. These materials are especially important owing to their bridging role between the world of conducting polymers and that of nanoparticles. For application of conducting polymers, knowing how these conducting polymer composites will affect the behaviour in an electric field is a long-standing problem and is of great importance. But very little is known about the dielectric properties of conducting polymer associated with the conducting mechanism. Dielectric spectroscopy has been found to be a valuable experimental tool for understanding the phenomenon of charge transport in conducting polymers. Low frequency conductivity and dielectric relaxation measurements especially have proven to be valuable in giving additional information on the conducting mechanism that d.c. conductivity measurement alone does not provide. In this work a new potential use of FA has been proposed. The high dielectric constant FA (Raghavendra et al 2002b) has been used for the synthesis of PPy/FA composites.

In our earlier work (Raghavendra et al 2003), we carried out studies on the synthesis, characterization and low frequency a.c. conduction of polyaniline-fly ash composites. In the present work, we report the study on morphology and conducting properties of PPy/FA composites, synthesized by chemical oxidation process. The study shows better utility of fly ash, in order to tailor electrical properties of PPy/FA composites.

\section{Experimental}

The AR grade pyrrole was purified by distillation under reduced pressure. A fine fresh clean and pure FA powder was collected from the Raichur Thermal Power Station, Raichur, India. The cenosphere type FA was grounded and sintered at $600^{\circ} \mathrm{C}$ to remove moisture content and some dust impurities present. Chemically, the FA was silica to an extent of $55-70 \%$ followed by alumina, 10 $18 \%$, iron oxide, 6-20\% and lime magnesia and alkalies varied between 1 and 5\% each. FA also contains elements like $\mathrm{Cu}, \mathrm{Pb}, \mathrm{Cd}, \mathrm{Ag}, \mathrm{Mn}, \mathrm{Ti}, \mathrm{Na}, \mathrm{Mo}, \mathrm{S}, \mathrm{P}, \mathrm{Zn}$ and $\mathrm{Cl}$ in different concentrations (Volgel 1969).

$0.03 \mathrm{M}$ of distilled pyrrole was added to the solution of $0.06 \mathrm{M}$ of ammoniumpersulfate $\left[\left(\mathrm{NH}_{4}\right)_{2} \mathrm{~S}_{2} \mathrm{O}_{8}\right]$ and this reaction mixture was stirred continuously at a constant temperature $\left(5^{\circ} \mathrm{C}\right)$ to obtain polypyrrole. To this reaction mixture, varied weight per cent of fly ash powder $(10,20$, 30, 40 and 50) was added to form polypyrrole-fly ash composites. The obtained product was filtered and washed thoroughly with methanol $\left(\mathrm{CH}_{3} \mathrm{OH}\right)$ and the sample was dried under vacuum for more than $24 \mathrm{~h}$ at room temperature.
The obtained composites were pressed in the form of circular pellets of $1 \mathrm{~cm}$ diameter. The a.c. conductivity measurements on these composites were made using the conducting silver paste as electrodes on both sides. A.c. conductivity measurements were carried out at room temperature over the frequency range $100 \mathrm{~Hz}-400 \mathrm{MHz}$ using the Hewlett-Packard impedance analyser 4192-A model. The d.c. conductivity of these composites was studied by varying the temperature from $40-200^{\circ} \mathrm{C}$ by applying constant voltage and measuring the current through the sample at different temperatures. The SEM images of polypyrrole-fly ash composite (50 wt\%) was investigated using Philips XL 30 ESEM Scanning Electron Microscope. The X-ray diffraction patterns of the samples in this present case were recorded on Philips $\mathrm{X}$ ray diffractometer using $\mathrm{CuK}_{\alpha}$ radiation $(\lambda=1.5406 \AA)$. The diffractograms were recorded in terms of $2 \theta$ in the range $20^{\circ}-80^{\circ}$ with a scanning rate of $2 \% \mathrm{~min}$. The IR spectra of all the samples were recorded on a Perkin Elmer (model 783) IR spectrometer in $\mathrm{KBr}$ medium at room temperature. For recording IR spectra, powders were mixed with $\mathrm{KBr}$ in the ratio $1: 25$ by weight to ensure uniform dispersion in $\mathrm{KBr}$ pellets. The mixed powders were then pressed in a cylindrical die to obtain clean discs of $\sim 1 \mathrm{~mm}$ thickness. The characterization of polypyrrole and its composites by spectroscopic methods is important, as it gives information not only about various molecular-level interactions but also on the type of charge carriers.

Fly ash is a finely divided amorphous powder with the particle size ranging from $150 \mathrm{~nm}-120 \mu \mathrm{m}$. It is abrasive and refractory in nature. In the present study the fly ash employed is of cenosphere type, collected from the Raichur Thermal Power Station, Raichur, India. Qualitative analysis of chemical composition of fly ash shows the presence of $\mathrm{SiO}_{2}(52-66 \%), \mathrm{Fe}(6-8 \%), \mathrm{Al}_{2} \mathrm{O}_{3}(21-$ $27 \%), \mathrm{Ca}(6 \%)$ and $\mathrm{Ti}, \mathrm{P}, \mathrm{Mg}, \mathrm{Na}, \mathrm{S}, \mathrm{K}(<1 \%)$.

\section{Results and discussion}

Figure 1 represents the plot of wt\% variation of fly ash with a.c. conductivity at different frequencies. From the plot, it is seen that a.c. conductivity is almost constant up to $30 \mathrm{wt} \%$ of fly ash and increases thereafter reaching a maximum at $40 \mathrm{wt} \%$ of fly ash and decreases further. At $40 \mathrm{wt} \%$ of fly ash, the values of a.c. conductivity are maximum because of the formation of excess charge carriers (polarons). In this particular weight percentage of PPy/FA composites, polarization due to hopping conduction dominates. The decrease in conductivity values may be attributed to the presence of fly ash particles of larger dimensions, which makes the hopping of charge carriers between the favourable sites more difficult.

Figure 2 shows the d.c. conductivity behaviour of polypyrrole fly ash composites from $40-200^{\circ} \mathrm{C}$. It is 


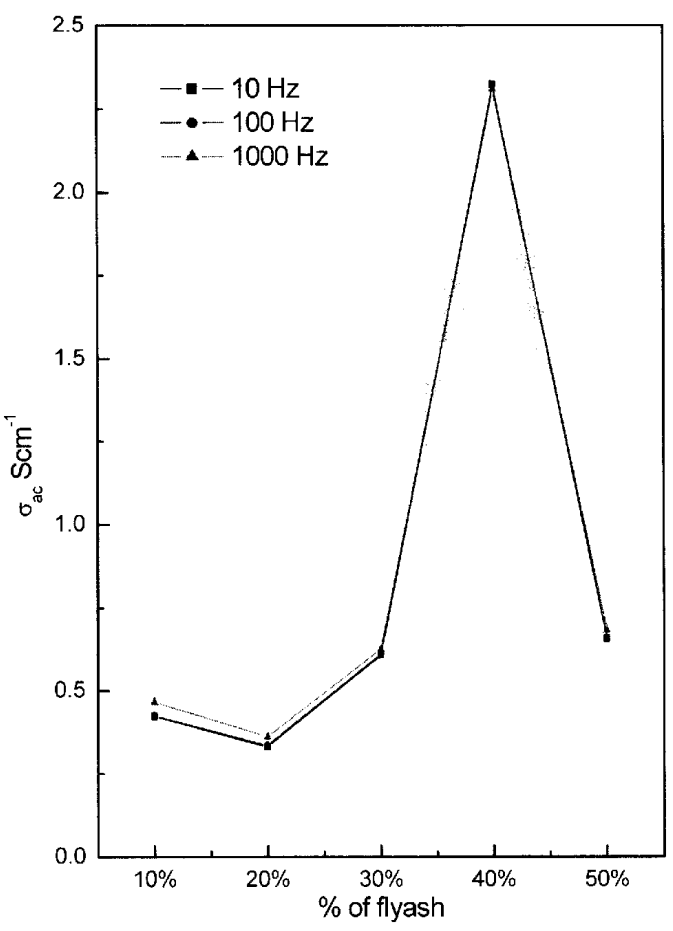

Figure 1. Variation of $\sigma_{\text {a.c. }}$ as a function of weight $\%$ of fly ash at different frequencies.

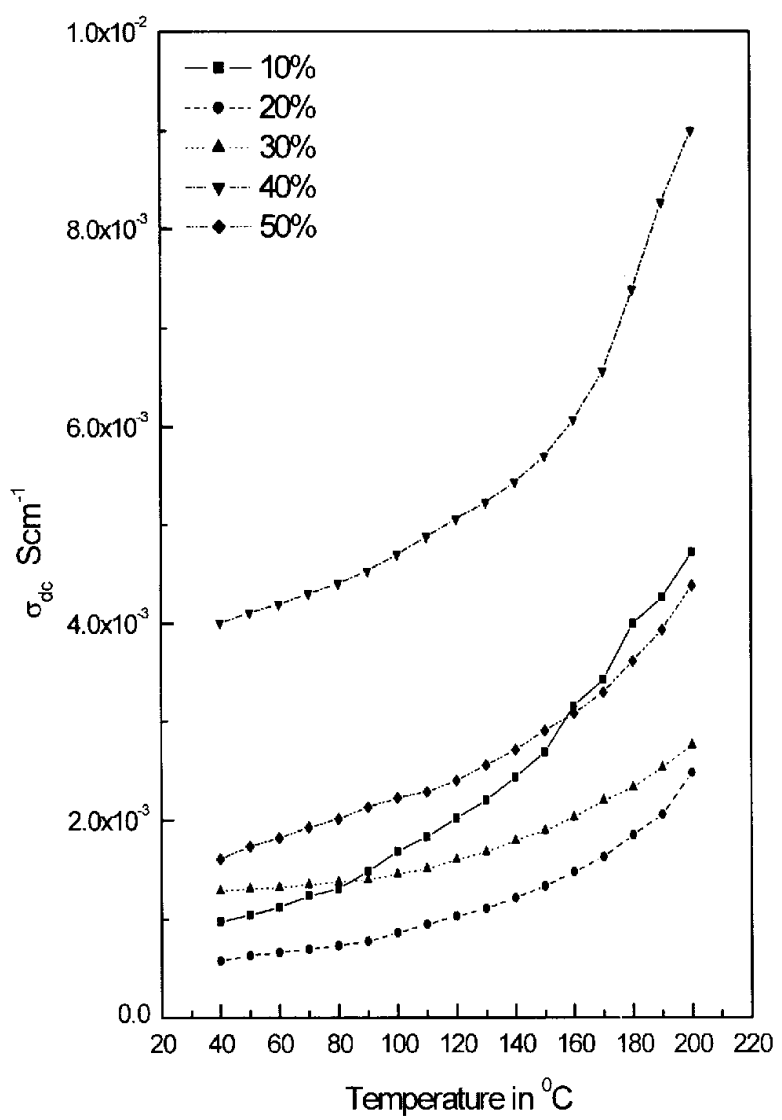

Figure 2. Variation of $\sigma_{\text {d.c. }}$ as a function of temperature for polypyrrole-fly ash composites. observed from the graph, that the conductivity of all the samples increases as the temperature increases and reaches maximum at $40 \mathrm{wt} \%$ of fly ash. These composites show negative temperature coefficient of resistance with temperature.

Figure 3 shows the SEM micrograph of polypyrrolefly ash composite (50 wt\% of fly ash). A very high magnification reveals the homogeneous distribution of fly ash (cenosphere) particles. It is seen from the micrograph that cluster and granular structure of polypyrrole is maintained even after the addition of fly ash in polypyrrole. Hence, a network of fly ash and granular polypyrrole has been formed in case of composites.

The IR spectra of polypyrrole-fly ash composite is shown in figure 4 . In the presence of fly ash the composite exhibits new absorption peaks distinctly at 1690, 1548, 1306, 1190, 1040, 910, 801, 678 and $550 \mathrm{~cm}^{-1}$ which are assignable to the presence of various metal oxides in the composite (Raghavendra et al 2002a). The spectra show that the absorption frequencies are slightly shifted towards lower side due to the weak Van der Waals forces.

Figure 5 shows the X-ray diffraction patterns of polypyrrole-fly ash composites (50 wt\% of fly ash in polypyrrole). Careful analysis of X-ray diffractogram of polypyrrole-fly ash composite suggests that it exhibits semi-crystalline behaviour. No structural change has been observed in fly ash due to its dispersion in polymerization of reaction of polypyrrole.

\section{Conclusions}

With more than 100 million tonnes of fly ash produced in India, use of fly ash for the preparation of polypyrrolefly ash composites will in no way help in its bulk utilization. Still the authors have made an effort towards the

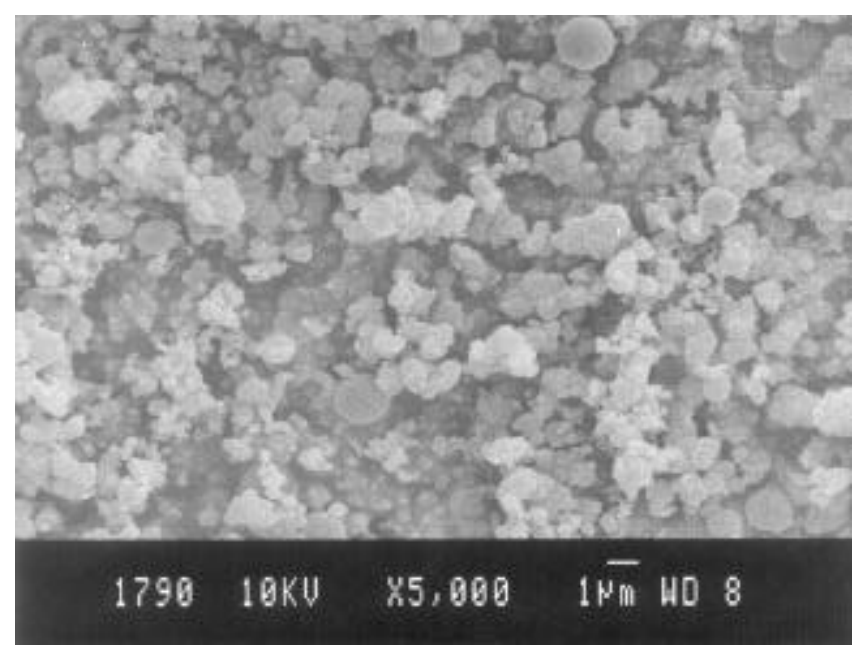

Figure 3. SEM micrograph of polypyrrole-fly ash composites (50 wt\% of fly ash). 


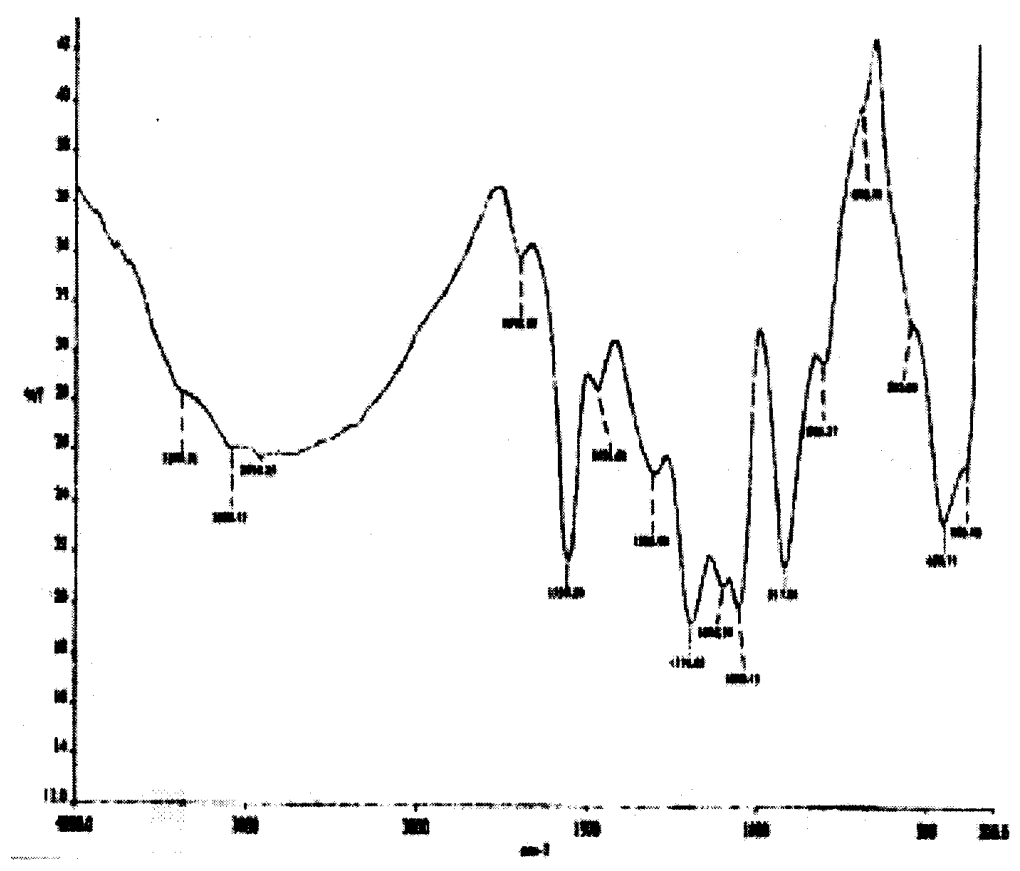

Figure 4. Infrared spectroscopy of polypyrrole-fly ash composites (50 wt $\%$ of fly ash).

worth

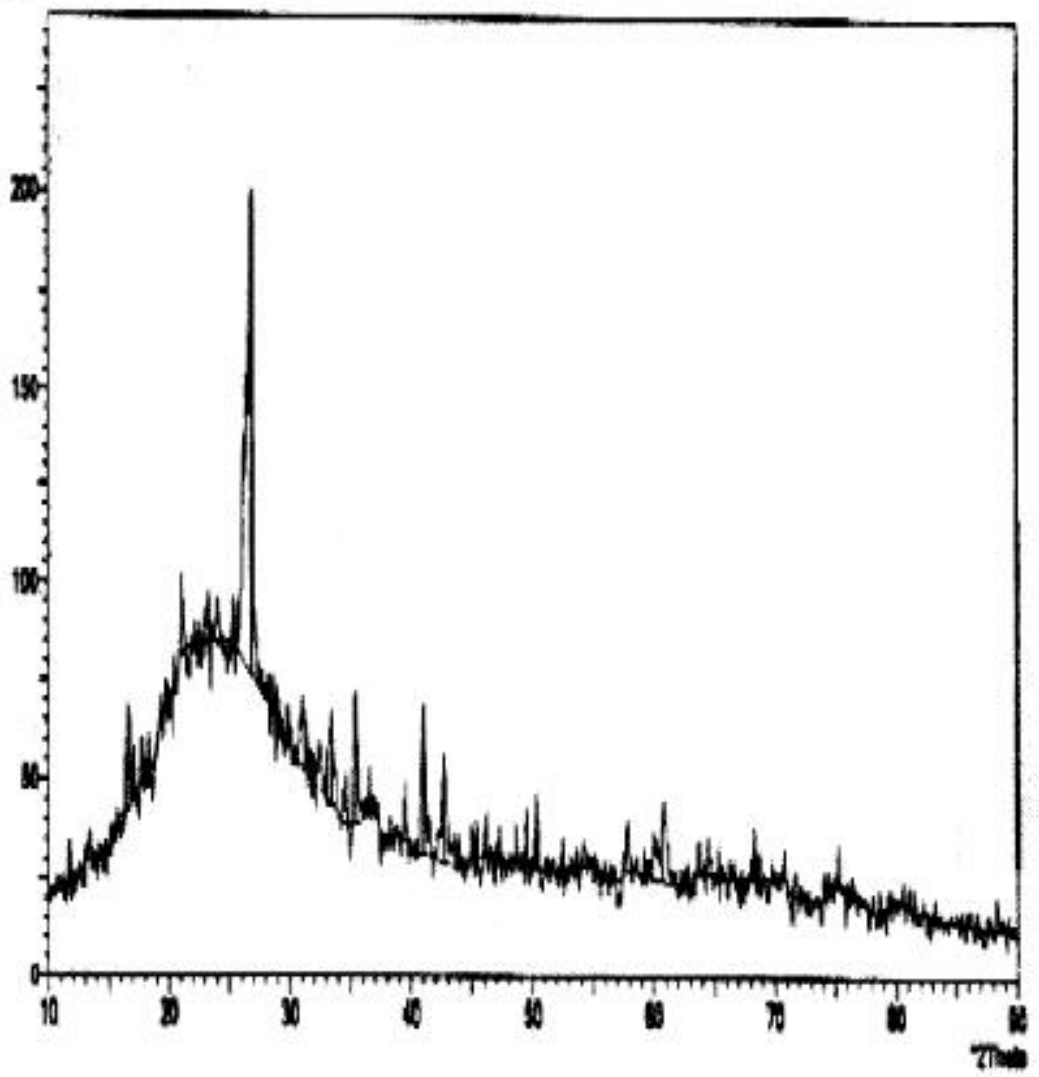

Figure 5. X-ray spectroscopy of polypyrrole-fly ash composites (50 wt $\%$ of fly ash). 
better utility of fly ash by synthesizing polypyrrole-fly ash composites. Detailed characterization of these composites were successfully carried out through XRD, SEM and IR. The results of both a.c. and d.c. conductivity show a strong dependence on the weight per cent of fly ash in polypyrrole. Further in depth study is required to understand the conductivity mechanism in these composites using theoretical simulation.

\section{Acknowledgements}

The authors would like to acknowledge Mr M Revanasiddappa, GM Institute of Technology, Davangere, for the discussion in synthesis part and Mr S C Raghavendra, Bhalki Rural College of Engineering, Bhalki, for better understanding of the chemical analysis of fly ash.

\section{References}

Cumpston B, Shadman F and Risbud S 1992 J. Mater. Sci. 27 1781
Kivelson S 1981 Phys. Rev. Lett. 461344

LaRosa J L, Kwan S and Grutzeek M W 1992 J. Am. Ceram. Soc. 751574

Meadea S and Armes S P 1995 Chem. Mater. 7171

Mohammadi A, Lundstrom I, Inganas O and Salaneck W R 1993 Polymer 31395

Niwa O and Tamamura T 1984 J. Chem. Soc. Chem. Commun. 817

Ojha K and Pradhan N C 2001 Indian J. Engg. Mater. Sci. 8100

Omastova M 1993 Synth. Met. 53227

Raghavendra S C, Raibagkar R L and Kulkarni A B 2002a Bull. Mater. Sci. 2537

Raghavendra S C, Raibagkar R L and Kulkarni A B 2002b Indian J. Pure \& Appl. Phys. 40367

Raghavendra S C, Syed Khasim, Revanasiddappa M, Ambika Prasad M V N and Kulkarni A B 2003 Bull. Mater. Sci. 26733

Sahu K C 1994 Power plant pollution: Cost of coal combustion, survey of the Environment, The Hindu 47

Sampathkumar N N, Umarji A M and Chandrashekar B K 1995 Mater. Res. Bull. 301107

Singh R, Tandon R P, Panwar V S and Chandra S 1991 J. Appl. Phys. 642504

Volgel A I 1969 A text book of quantitative inorganic analysis (London: Longman) 